\title{
DYNAMICS OF ENERGY TRANSPORT IN TERNARY MOLECULAR SOLIDS. II. TIME EVOLUTION OF NAPHTHALENE FLUORESCENCE. *
}

\author{
Panos ARGYRAKIS ** and Raoul KOPELMAN \\ Department of Chemistry. University of Michigan. Ann Arbor. Michigan 48109. USA
}

Received 8 September 1982; in final form 14 March 1983

\begin{abstract}
The spectrally resolved time-evolution of free and trapped singlet excitons was obtained at liquid-helium temperature for ternary crystals of perdeuteronaphthalene/naphthalene/betamethylnaphthalene (host/guest/supertrap). The naphthalene guest (donor) concentration varied between 0.30 and 0.99 mole fraction. while the supertrap (acceptor) concentrations were $10^{-4}-10^{-5}$. At the lower guest concentrations ( 0.50 and below) the naphthalene-exciton decay time approaches the natural lifetime $(=122 \mathrm{~ns})$. At higher concentrations. the decay is much shorter and extremely non-exponential. This behavior is inconsistent with simple homogeneous kinetics schemes that use a time-independent rate constant for energy transport. Above the percolation concentration (0.60 naphthalene) we fitted the experimental results with a random-fight-kinetic model. incorporating correlated random walks on the percolating guest cluster. The best fit was obtained for a "coherence length" (mean free path) of $=10^{2}$ lattice units. These results are in good agreement with previous steady-state studies on the same samples, and seem to indicate a partial coherence of the exciton transport in both pure and substitutionally disordered crystals at these low temperatures.
\end{abstract}

\section{Introduction}

The characteristics of transport in disordered systems is a problem of current interest [1-8]. The transport or migration of optical excitations offers some simplifying features both theoretically, e.g. no long-range Coulomb interactions, and experimentally, e.g. the utilization of powerful laser excitation and detection techniques with high temporal, spatial and energy resolution. Recently there has been a significant increase in the number of theoretical papers on the transfer of excitation in disordered molecular aggregates [6-22]. We do not consider here the interesting problem of transfer from one donor to a set of randomly distributed acceptors [23,24], but only the case of donor-donor transfer and migration where the donors are randomly distributed and where the occurrence of donor-acceptor transfer is a rare (though im-

* Supported by NIH Grant No. 2 R01 NS08116-14 and NATO Grant No. SA 05RG 295/82.

** Permanent address: Department of Physics, University of Crete, Iraklion. Crete. Greece. portant) event. Experimentally we produced a system that has random substitutional disorder but positional and orientational order at the same time. Moreover. for most practical purposes our samples were perfect single crystals of high physical and chemical quality.

Much of the recent interest in exciton-transport problems has been associated with the phenomena of critical concentrations [3.5.19] (characterizing significant enhancement of migration). To explain these "transitions" of transport, Anderson localization [6.27], percolation [25.26.28], and other models [7.8] have been suggested. involving different physical pictures of the exciton transport. Here we describe experiments that are only marginally related to the question of the Anderson transition. However, these studies are of much relevance to the topics of exciton diffusion (dispersive [8] or percolative [29,30]). exciton coherence and exciton-phonon coupling. Furthermore, a quantitative kinetic model is suggested where the controlling factor is a form of "heterogeneous" diffusion (percolation), which may have some wider applica- 
tions, such as for migration in photosynthetic systems [31].

We have recently described in detail $[32,33]$ a model based on a Monte Carlo method that attacks the problem of energy transport utilizing simulations on large molecular lattices in the computer memory. This model is quite versatile and enables one to go beyond the common analytical versions of random walks [34]. With this model we can treat practically any dimensionality and any combination of interactions for any number of components at any giver concentration. In addition, our model includes directional correlation and memory of the motion. The parameter we often measure is the extent of energy transfer given by the number of molecular sites that have carried energy at one time or another during the "experiment". This number, divided by the number of "steps", gives the efficiency, $\epsilon$. We reported $[35,36]$ the efficiency as a function of time for various parameters and over a range of several exciton lifetimes.

We used the above model, in conjunction with a series of steady-state experiments [33] (paper I of this series) on the naphthalene system $\left(C_{10} H_{8} / C_{10} D_{8} /\right.$ betamethylnaphthalene- $d_{10}$, guest/host/supertrap), to study some details of excitonic energy transfer. This pointed towards the existence of some "coherence" at liquid-helium temperatures for this system. In the present study, a continuation of the previous work, we investigated the time-dependence of the system. We developed a kinetic scheme starting from the differential equations for the concentrations of excited states in our system. The rate coefficients in these equations (which are time-dependent) were related to the above-mentioned efficiency of transport model in a formalism discussed in section 3 of this paper. These equations were solved numerically in section 4 for the excitation populations with only one adjustable parameter, the coherence length $l$, and the various solutions were compared to our experimental results. Our conclusions are given in section 5 , and pertain to exciton localization and the partial coherence of the energy transport in substitutionally disordered and pure crystals at low temperatures.

\section{Experimental}

Most experimental details were described in paper I (of this series [33]). At very narrow-band excitation (of the order of $1 \mathrm{~cm}^{-1}$ ), the output of a nitrogen laser pumped dye laser (Molectron DL 400) was used as the exciting light source, frequency doubled. Since the laser light is tunable, any component or energy level can be selectively excited, even impurity molecules or the BMN (betamethylnaphthalene- $d_{10}$ ) molecules. A typical excitation frequency was $32000 \mathrm{~cm}^{-1}$, which is well above the naphthalene " $0-0$ " energy level of the first excited singlet state ${ }^{1} B_{2 u}$. We occasionally varied this frequency down to $\approx 31280 \mathrm{~cm}^{-1}$. For a $C_{\mathrm{g}}=\mathbf{0 . 5 0}$ concentration crystal the bottom of the exciton band is $\approx 31450 \mathrm{~cm}^{-1}$. We could thus directly establish the bottom of the band, and we found it to be in good agreement with the values obtained by other methods [37,38]. The intensity of the exciting light was also varied by several factors of two to observe any effects on the emitted signal. This was done quantitatively using a polarizer, by changing the angle of polarization. The results are given in section 4.

The spectra were taken at 1.8 and $4.2 \mathrm{~K}$ using the same fluorescence bands as described earlier: the "0-512" for $\mathrm{C}_{10} \mathrm{H}_{8}$ and the "0-0" for BMN. $\mathrm{BMN}$ is a monomer due to its low concentration, and has a relatively high intensity. When the BMN contribution to the total intensity was low its time-evolution spectrum was very noisy or sometimes non-recordable. This was because at high $\mathrm{C}_{10} \mathrm{H}_{8}$ concentrations, even though the transfer is very efficient, the BMN concentration is very small. The small concentration is because that BMN comes as a "natural" (perdeuterated) impurity in the $\mathrm{C}_{10} \mathrm{D}_{8}$ host [33]. In all cases the $\mathrm{C}_{10} \mathrm{H}_{8}$ intensity was strong enough for the time-resolved spectra to be recorded.

\section{Kinetic formalism}

We assume that all excitation is generated on the guest lattice sites. Experimentally, the excitation initially localized on the host will decay rapidly to the guest, and since the concentration of BMN 
is much lower than that of naphthalene, we can neglect any direct excitation of BMN. The BMN fluorescence is proportional to [BMN], the number of excited BMN molecules. Also, the number of excited guest molecules is $[N]$, and is assumed to be small compared to the BMN and $\mathrm{N}$ concentrations (however, see below). We neglect collisions between excitons and saturation of the BMN species. The energy transfer can then be thought of as the migration of these guest singlet excitons. and their spread throughout the lattice, followed either by direct monomolecular decay or by trapping by the BMN (and decay from there). Thus, we arrive at the following kinetic equations that describe the rate of change of the $\mathrm{N}$ and $\mathrm{BMN}$ exciton concentrations $[1,39]$ :

$$
\begin{aligned}
& \mathrm{d}[\mathrm{N}] / \mathrm{d} t=-k_{\mathrm{N}}[\mathrm{N}]-k(t)[\mathrm{N}], \\
& \mathrm{d}[\mathrm{BMN}] / \mathrm{d} t=-k_{\mathrm{B}}[\mathrm{BMN}]+k(t)[\mathrm{N}] .
\end{aligned}
$$

Here $k_{\mathrm{N}}$ and $k_{\mathrm{B}}$ are the experimental (monomolecular) decay rates for isolated naphthalene and $B M N$, respectively, and include radiative and non-radiative processes, but no intermolecular energy transfer. The time-dependent coefficient $k(t)$ describes the energy transfer rate at time $t . k(t)$ is also related to the probability that a BMN supertrap molecule will have trapped a naphthalene exciton at time $t$. The solutions of these equations can then be related to the time resolved spectra. In these equations, $k_{\mathrm{N}}$ and $k_{\mathrm{B}}$ are independent and well-known quantities, and $k(t)$ is the only system-dependent quantity.

The above kinetic scheme was previously postulated for binary systems [1]. We propose a new quantitative approach for both binary and ternary systems. For ternary systems we present a new model based on the percolation approach. In particular, this involves the definition of the time-dependent rate coefficient, $k(t)$.

It has been shown [40] that the total probability for trapping by $\mathrm{BMN}$ is given as

$P=P_{\infty}\left[1-\exp \left(-S_{n} \gamma C_{s}^{\prime}\right)\right], \quad C_{\mathrm{s}}^{\prime}=C_{\mathrm{s}} / C_{\mathrm{B}}$.

Here $P_{\infty}$ is the percolation probability [40], $C_{s}$ is the supertrap concentration (mole fraction), and $\gamma$ is its trapping efficiency. Introducing the quantity $\epsilon$ ( $\epsilon=S_{n} / n$, where $S_{n}$ is the number of sites visited in an $n$-step walk) one gets

$P=P_{\infty}\left[1-\exp \left(\epsilon n \gamma C_{s}^{\prime}\right)\right]$.

Using $a=C_{s}^{\prime} \gamma n / t$, where $t$ is the time, we finally obtain

$P=P_{\infty}[1-\exp (-a \epsilon t)]$.

The change of probability per unit time is then $\mathrm{d} P / \mathrm{d} t$ and is equal to

$\mathrm{d} P / \mathrm{d} t=P_{x} \mathrm{e}^{-u t} a(\epsilon+t \mathrm{~d} \epsilon / \mathrm{d} t)$.

This quantity is proportional to $k(t)$ and it can be shown (see appendix $A$ ) that the "proportionality constant" is equal to $1 /(1-P)$. Therefore we have

$k(t)=\frac{P_{x} a \mathrm{c}^{-\alpha e t}(\epsilon+t \mathrm{~d} \epsilon / \mathrm{d} t)}{1-P_{x \in}\left(1-\mathrm{e}^{-\alpha \epsilon t}\right)}$.

We emphasize that $\epsilon$ is a function of $C_{\mathrm{g}}, t . l$. and the topology. For $C_{g} \geqslant 0.70$. for the square lattice topology. $P_{x} \approx 1.0$ and eq. (7) becomes

$k(t)=a(\epsilon+r \mathrm{~d} \epsilon / \mathrm{d} t)$.

This form is rigorously true for a binary system. We saw above that $k(t)$ depends on the quantity $\epsilon$. and also depends (via $a$ ) on $\gamma$ and $C_{5}$. Both $\gamma$ and $C_{\mathrm{s}}$ are properties of the system at hand. so we are now in a position to calculate $k(t)$. The differential equations now become

$$
\begin{aligned}
& \mathrm{d}[\mathrm{N}] / \mathrm{d} t=-k_{N}[\mathrm{~N}]-a(\epsilon+t \mathrm{~d} \epsilon / \mathrm{d} t)[\mathrm{N}] . \\
& \mathrm{d}[\mathrm{BMN}] / \mathrm{d} t=-k_{\mathrm{B}}[\mathrm{BMN}]+a(\epsilon+t \mathrm{~d} \epsilon / \mathrm{d} t)[\mathrm{N}]
\end{aligned}
$$

We seek solutions to these coupled equations. It has been shown [35] that $\epsilon$ follow's an approximately exponential decay. We therefore fit the simulation results of $\epsilon$ to an exponential equation to get the best fit (or alternatively. we fit $\log \epsilon$ to a linear equation). Thus

$$
\begin{aligned}
& \epsilon=\mathrm{e}^{b t+t} . \\
& \log \epsilon=b t+A . \\
& \mathrm{d} \epsilon / \mathrm{d} t=b \epsilon, \\
& k(t)=a(\epsilon+t \mathrm{~d} \epsilon / \mathrm{d} t)=a \epsilon(1+b t) .
\end{aligned}
$$

This process predicts $k(t)$ rather satisfactorily 
for most of the $C_{\mathrm{g}}$ concentration range, but not so well close to the critical percolation concentration $C_{c}$. At this point the approximation in eq. (11) may give an error up to $50 \%$ in the fitted values of $\epsilon$, and therefore it is advisable to use the full eq. 8 to calculate $k(t)$. We solve eq. (8) numerically by calculating the slope $d f / d t$ at each $\Delta t$ interval. The resulting function $k(t)$ contains more noise than the fitted $k(t)$ of eq. (14) due to the inherent statistical fluctuations in the slopes $\mathrm{d} \epsilon / \mathrm{d} t$, but is a much better approximation than eq. (14), at least for the concentration region close to $P_{c}$. In fig. 1 a plot is shown for $k(t)$ versus $t$. for a typical calculation. As expected, $k(t)$ is not a linear function of time.

Eq. (9) now becomes

$\mathrm{d}[\mathrm{N}] / \mathrm{d} t=-k_{\mathrm{N}}[\mathrm{N}]-a \epsilon(1+b t)[\mathrm{N}]$.

and after separating variables and integrating:

$\log [\mathrm{N}]=-\int\left[k_{n}+a \epsilon(1+b t)\right] \mathrm{d} t$
$[\mathrm{~N}]=\exp \left(-\int\left[k_{N}+a \epsilon(1+b t)\right] \mathrm{d} t\right)$.

The integral on the right-hand side is then evaluated numerically. Eq. (2) now becomes

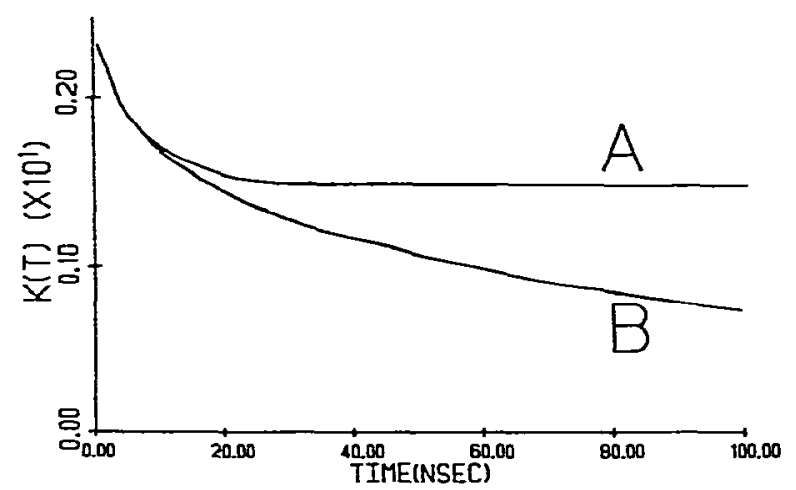

Fig. 1. Time-dependent rate-"constant” behavior. The time-dependent rate constant $k(t)$ is plotted as a function of time. according to eq. (9). The solution is given for a concentration $C_{\mathrm{s}}=\mathbf{0 . 7 0}$ for two coherence lengths, $l=1$ (A), and $l=10$ (gaussian distribution with standard deviation $\sigma=3.0$, curve B). The units of $k(t)$ are sites visited/ns. The two curves $A$ and B are the smoothed $k(t)$, all noise being averaged out for clearness.
$\mathrm{d}[\mathrm{BMN}] / \mathrm{d} t+k_{\mathrm{B}}[\mathrm{BMN}]=k(t)[\mathrm{N}]$.

The general solution of this equation can also be evaluated numerically since

$$
\begin{aligned}
& {[\mathrm{BMN}](t)=\mathrm{e}^{-k_{\mathrm{B}} t} \int \mathrm{e}^{k_{\mathrm{B}} t} k(t)[\mathrm{N}](t) \mathrm{dt}} \\
& {[\mathrm{BMN}](t)=\mathrm{e}^{-k_{\mathrm{B}} t} \int \mathrm{e}^{k_{\mathrm{B}} t} a \mathrm{e}^{t t+a}(1+b t)[\mathrm{N}] \mathrm{d} t .}
\end{aligned}
$$

Similar but more complex solutions apply when $P_{\infty} \neq 1$. using the full eq. (7). The latter were actually used numerically for the work reported below.

A few comments are in order at this point. The solutions in eqs. (16) and (20) depend on $P_{\curvearrowright}, k(t)$. $a, b$, and time. Here $a$ and $b$ are constants which depend on the efficiency $\epsilon$, the supertrap concentration $C_{s}$. and the ratio $n / t$, which is the number of steps per unit time (but not the absolute number of steps), but they ( $a$ and $b$ ) do not depend on time or the coherence $l$. Similarly $k(r)$ depends on $a$ and $b$ via eq. (7). and it is also a function of time. $P_{\infty}$ is a static property of a given lattice, but has no dependence upon any energytransfer mechanism (it depends only on the interaction topology and the guest concentrations). Thus the only quantities reflecting the dynamical nature of the system are $\epsilon$ and the time $(t)$. Notice that the information about the coherence length $l$ is incorporated in $\epsilon$. This is so because $\epsilon$ depends on $S_{n}$, and $S_{n}$ is a strong function of $l$. It was found $[35,36]$ that large $l$ values increase $S_{n}$ at the high $C_{\mathrm{g}}$ range, but decrease $S_{n}$ at $C_{\mathrm{g}}$ close to the critical percolation concentration. We can use simple numerical solutions for $\epsilon$, which are now available in the literature $[35,41]$. These numerical results for $\epsilon$ can be checked against other work (using the generating function method or the elliptic integral method) only for the pure crystal limit, and we found them to be in satisfactory agreement $[59,60]$. We thus solved the kinetic equations in terms of simple parameters that we know how to calculate.

\section{Results}

We now proceed to derive the detailed solutions 
as discussed in the previous section. using the proper experimental boundary conditions. We first assume an instantaneous creation of the naphthalene excitons, so that. after normalizing. $[N]=$ 1.00 at time $t=0$. This assumption is good if we consider that it probably takes only a few picoseconds for the excitation to be localized on some naphthalene guest site (compared to the experimental resolution of a few nanoseconds). Similarly, $[\mathrm{BMN}]=0$ at time $t=0$. because we assume no direct excitation of the supertrap (because of its low concentration), and it takes. on the average. a considerable number of steps before a BMN site will be visited.

Experimentally the above boundary conditions are good only in principle. i.e. only when the excitation source is a delta function that creates excitons in a time shorter than the order of one jump. However, in practice this is not the case. Our laser is a pulsed light source with each pulse having an approximately gaussian width at halfheight of $\approx 5$ ns (after doubling). This is of comparable magnitude with the exciton activity times. and must be taken into account. $A$ direct way of doing this would be to record a blank laser signal and then "subtract it out" from the experimental signal, i.e. perform a deconvolution of the two signals. This operation, however, presents serious difficulties because, by nature. the experimental signal contains random noise which is amplified in the deconvolution process (which in effect is a differentiation process). and the method thus fails in practice. If the functional form of the experimental signal is known the problem of random noise can be easily eliminated and the deconvolution car be done, but as we noted in section 3 these solutions may turn out to be very complex functions whose form is not immediately known.

We resort to a second alternative: We perform a convolution of the calculated [N] and [BMN] signals with the laser signal (which in effect corresponds to an integration process). so as to make the predicted curves look experiment-like. and include exactly the same effects. This operation is somewhat complex, but feasible, and the details are shown in ref. [49]. For background discussion on convolution theory see refs. [50-55].

Because the excitation source has a relatively high intensity. the question arose whether exciton-exciton-interaction phenomena can be neglected. In some crystal systems. such as anthracene, the exciton-exciton-annihilation process is well-known [42]. Some information became available recently [43] for the naphthalene singlet. but only for high temperatures ( $77 \mathrm{~K}$ and above) and pure lattices. Recently, results for binary crystals appeared [44]. We tried a number of tests on this system. such as reducing the intensity of the incoming light by a factor of two. several times. We observed some differences orer the course of several runs. but the laser intensity. focusing on the crystal, clearness of the crystal. etc.. were not constant in day-to-day operations. and therefore we were not able to quantitatively determine any effects from these runs. Further tests on the same crystals [45]. using neutral density filters, showed no significant effect. For our kinetic scheme equations we used the data where further cutting down on the intensity produced no visible changes in the relative spectral intensities. hoping that at this point we had reduced all exciton-exciton interactions to a minimum. Speculating on our observations. we occasionally noticed that at high light intensities the guesi/trap emission ratio decreased. indicating that the $\mathrm{C}_{10} \mathrm{H}_{s}$ exciton concentration decreased. The probable cause is exciton-exciton fusion. The fusion effect was probably significant only at high $C_{\mp}(0.99)$. where the BMN concentration was the lowest. thus enhancing the fusion/trapping ratio. The collision rate constant for this process should be an interesting topic of study [46].

Our experimental data have a considerable uncertainty. It is not surprising that small variations in the supertrap concentration (even within the same crystal) could cause a doubling in the component lifetime (defined here as the time necessary for the intensity to drop down to $1 / \mathrm{e}$ of its peak value). For example, a change by a factor of 10 in $C_{.}$(from $10^{-5}$ to $10^{-7}$ ) causes a change of 0.60 in the probability for trapping (from 0.39 to 0.99 ). see fig. 2 of ref. [41]. However. the decay times for crystals from $C_{\mathrm{a}}=0.70$ to $C_{\mathrm{g}}=0.99$ range from $=12$ to 40 ns. which is a factor of 4 . Also. the calculated quantities show a similar variation. For example. for the $C_{g}=0.80$ crystal. for $C_{s}=1 \times$ 
$10^{-4}$ the calculated decay times are 27,16 , and 45 ns, while for $C_{\mathrm{s}}=1 \times 10^{-5}$ they are 100,85 , and $120 \mathrm{~ns}$, a factor of 5 (for coherence lengths $l=1$, 10 and 100, respectively). We used the average of all our decay measurements for each crystal. For the calculations we used the proper "average" supertrap concentration (see paper I). Below the critical percolation concentration the decay times get longer, approaching the natural lifetime of naphthalene. This happens because in this region we have mostly isolated naphthalene clusters of small size, with a low probability of containing any $\mathrm{BMN}$ sites. Therefore, the $\mathrm{C}_{10} \mathrm{H}_{8}$ excitons never get trapped, but instead "live" within these small clusters throughout their natural lifetime. The decay times in this region vary from $60 \mathrm{~ns}$ to $=120 \mathrm{~ns}$. The lifetime of neat naphthalene is $\approx 120 \mathrm{~ns}$. A summary of all lifetimes is given in table 1 . Similar results for this region of $C_{g}$, with higher $C_{s}$, were obtained by Parson and Kopelman [29].

The experimental time decays of fig. 3 are more complex than simple exponentials, as we can easily see when we plot the logarithmic spectra. This situation is different from the work of Parson and Kopelman [29], where they observed exponential behavior in the guest lifetimes throughout the concentration range. The reason for this is that their transport is very fast, due to the presence of 1-2 orders of magnitude more supertrap BMN. Their actual nanosecond-domain kinetics is governed by eqs. (1) and (2) in the limit of $k(t) \rightarrow$ constant, with $k(t) \rightarrow 0$ for the lowest $(0.42)$ guest con-

Table 1

Experimental lifetimes (ns).

\begin{tabular}{lccccc}
\hline$C_{8}$ & $1.8 \mathrm{~K}$ & & & \multicolumn{2}{l}{$4.2 \mathrm{~K}$} \\
\cline { 2 - 3 } \cline { 5 - 6 } & $\mathrm{C}_{10} \mathrm{H}_{8}$ & $\mathrm{BMN}$ & & $\mathrm{C}_{10} \mathrm{H}_{8}$ & $\mathrm{BMN}$ \\
\hline 0.99 & 40 & & 23 & \\
0.95 & 12 & 35 & 12 & 42 \\
0.90 & 15 & 40 & & 20 & 45 \\
0.85 & 18 & 42 & & 15 & 50 \\
0.80 & 36 & 60 & & 16 & 44 \\
0.70 & 34 & 46 & 29 & 58 \\
0.60 & 36 & 44 & 25 & 45 \\
0.50 & 120 & 71 & 122 & 81 \\
0.40 & 80 & 57 & 83 & 67 \\
0.30 & & 86 & 95 & 76 \\
\hline
\end{tabular}

centration (following $k(t) \rightarrow \infty$ in the picosecond domain, see appendix A). In our case it is not surprising to find different results due to the lower concentration of BMN which makes the nanosecond-domain transport dependent on larger values of $k(t)$ for longer times.

The experimental spectra were all recorded at 1.8 and $4.2 \mathrm{~K}$. We did not observe any consistent trend between the two temperatures, such as we observed in the steady-state spectra [33]. A typical difference is shown in fig. 2 , where we plotted the spectra for both temperatures for the 0.70 mole fraction guest concentration crystal. We, therefore, averaged the integrated results of the two temperatures ${ }^{\ddagger}$.

* We cannot claim any definite switch in temperature effects as was recently observed for the delayed fluorescence (i.e. triplet transport) [61] and as was observed for the neat crystal fluorescence (singlet) experiments (with protonated BMN supertrap) at higher temperatures [62].

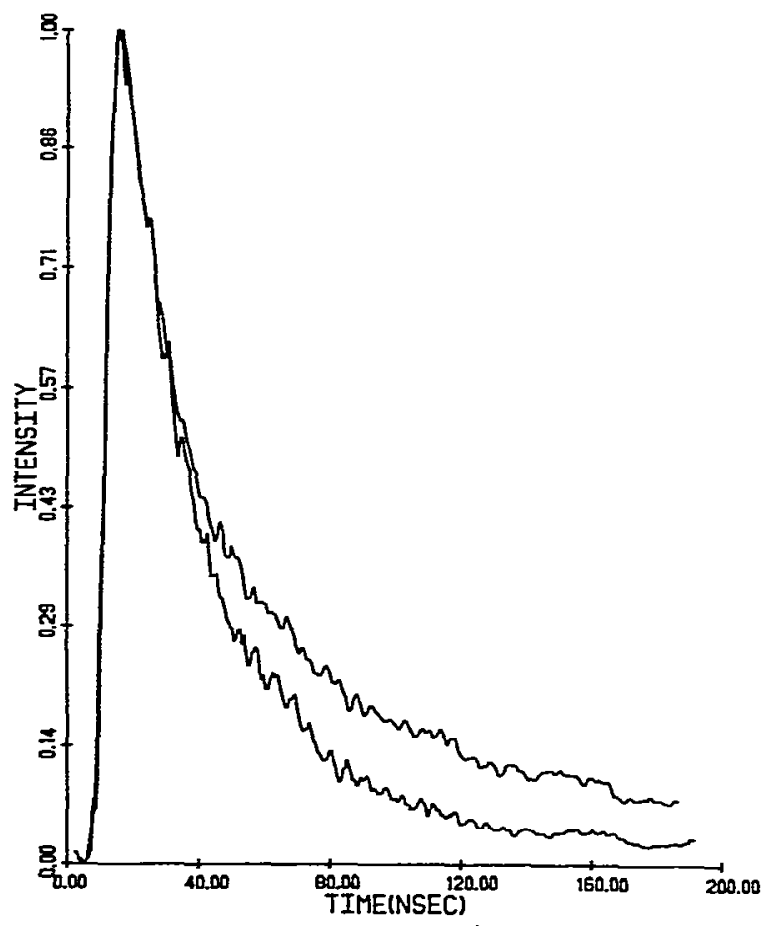

Fig. 2. Experimental time-evolution spectra for two different temperatures. The time-evolution spectra are shown for the 0.70 guest crystal, at two different temperatures, $1.8 \mathrm{~K}$ (top), and $4.2 \mathrm{~K}$ (bottom). The naphthalene emission was monitored in this case. In this crystal $C_{s}=1 \times 10^{-4}$. 

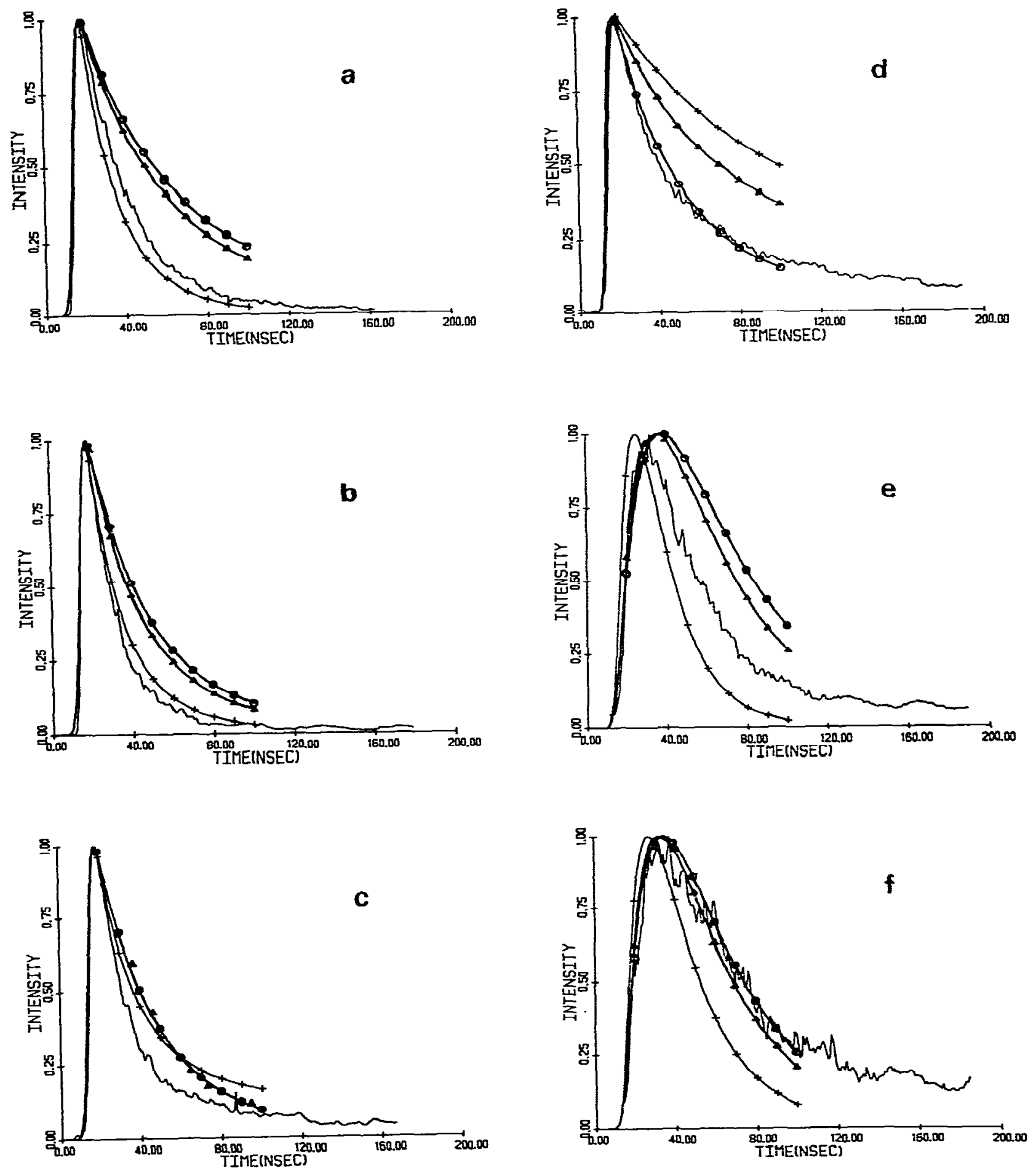

Fig. 3. For caption see next page- 

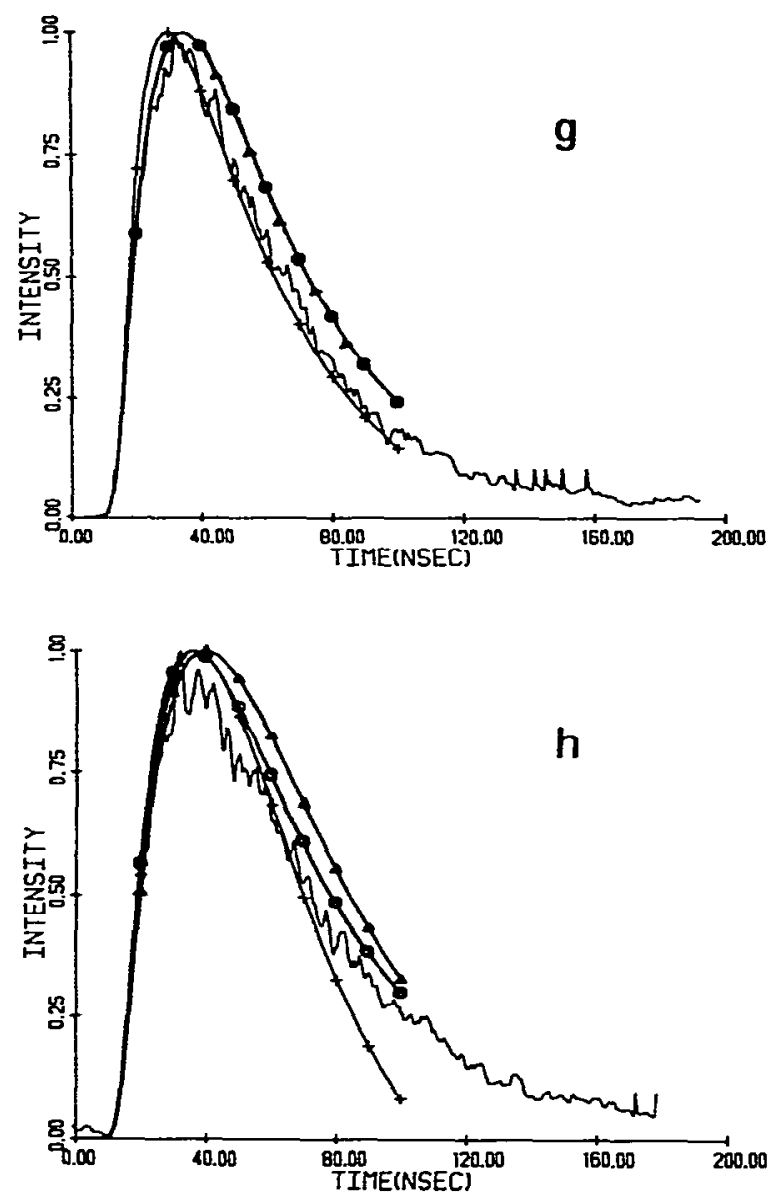

Fig. 3. Experimental and calculated time-evolution spectra. Plots of the solutions of the differential equations together with the experimental curves for several different guest concentrations, as marked. The naphthalene guest emission was moni-
The effects of coherent versus incoherent walks can be seen in the three calculated curves plotted in fig. 3 , where $l=1,10$, and 100 . Each one predicts a different time evolution behavior. owing to the difference in the $S_{n}$ values.

The most obvious conclusion from these curves is that at high $C_{\mathrm{g}}$. where the $l=1$ curve shows a considerably slower decay than the $l=10$ and $l=100$ curves. the experimental curve is close to the $l=10$ and $l=100$ curves, i.e. it shows a comparatively fast decay. At intermediate $C_{\mathrm{g}}$, about $C_{g}=0.70$, the $l=1$ curve shifts and exhibits the fastest decay, while the coherent cases $(l=10$ and $l=100$ ) show a slower decay, but still in agreement with the experiment.

In order to get a quantitative measure of the appropriateness of this picture we integrated the areas under the decay curves and compared the integrals. Table 2 shows the calculated integrals at 25,50 and $100 \mathrm{~ns}$, together with the experimental integrals. when the naphthalene emission was monitored. This corresponds to measuring the total

tored: (a) $C_{\mathrm{g}}=0.95 . C_{\mathrm{s}}=1.7 \times 10^{-5}$; (b) $C_{\mathrm{g}}=0.85, C_{\mathrm{s}}=5.1 \times$ $10^{-5}$ : (c) $C_{\mathrm{g}}=0.80, C_{\mathrm{s}}=6.8 \times 10^{-5}$; (d) $C_{\mathrm{g}}=0.70, C_{\mathrm{s}}=1 \times$ $10^{-4}$. The BMN trap emission was monitored: (e) $C_{\mathrm{B}}=0.90$, $C_{\mathrm{s}}=3.4 \times 10^{-5} ;$ (f) $C_{\mathrm{g}}=0.85, C_{\mathrm{s}}=5.1 \times 10^{-5} ;$ (g) $C_{\mathrm{g}}=0.80$. $C_{s}=6.8 \times 10^{-5}$; (h) $C_{g}=0.70, C_{s}=1 \times 10^{-4}$. The following coherence lengths were used: $l=1$ (circles). $l=10$ (with standard deviation $\sigma=3.0$, triangles), and $l=100$ (with standard deviation $a=30.0$. crosses). The calculated intensities were adjusted using the factor $a^{\prime}$ as discussed in the text. For simplicity eq. (14) was used for the $k(t)$ calculation. since the error introduced is within our other uncertainties.

Table 2

Integrated experimental and calculated intensities - naphthalene

\begin{tabular}{|c|c|c|c|c|c|c|c|c|c|c|c|c|}
\hline \multirow[t]{2}{*}{$C_{\mathrm{B}}$} & \multicolumn{4}{|l|}{$25 \mathrm{~ns}$} & \multicolumn{4}{|l|}{$50 \mathrm{~ns}$} & \multicolumn{4}{|l|}{$100 \mathrm{~ns}$} \\
\hline & exp. & $l=1$ & $l=10$ & $l=100$ & exp. & $l=1$ & $l=10$ & $I=100$ & exp. & $l=1$ & $l=10$ & $I=100$ \\
\hline 0.99 & 10.2 & 11.0 & 11.0 & 11.0 & 24.2 & 32.0 & 31.0 & 31.1 & 37.7 & 61.3 & 56.8 & 57.0 \\
\hline 0.95 & 9.8 & 11.0 & 10.9 & 10.9 & 18.6 & 29.6 & 26.3 & 26.1 & 24.6 & 50.7 & 39.0 & 40.3 \\
\hline 0.90 & 9.9 & 10.9 & 10.7 & 10.7 & 17.3 & 27.7 & 22.8 & 23.8 & 19.7 & 43.7 & 29.5 & 32.3 \\
\hline 0.85 & 10.2 & 10.9 & 10.5 & 10.6 & 19.9 & 26.1 & 20.9 & 21.9 & 24.6 & 38.7 & 25.6 & 38.2 \\
\hline 0.80 & 11.6 & 10.8 & 10.6 & 10.9 & 24.3 & 25.6 & 21.4 & 27.7 & 34.3 & 36.9 & 26.4 & 47.2 \\
\hline 0.75 & & 10.8 & 10.8 & 11.0 & (25) & 25.5 & 24.2 & 30.8 & (35) & 36.8 & 32.9 & 59.1 \\
\hline 0.70 & 11.8 & 10.9 & 10.9 & 11.0 & 25.6 & 26.1 & 27.9 & 32.3 & 36.7 & 39.1 & 46.9 & 63.2 \\
\hline 0.65 & & 10.9 & 11.0 & 11.0 & $(25)$ & 27.6 & 31.3 & 32.1 & (36.7) & 44.1 & 59.7 & 62.8 \\
\hline 0.60 & 10.1 & 11.0 & 11.0 & 11.0 & 25.0 & 29.8 & 32.6 & 32.6 & 36.8 & 52.8 & 64.0 & 64.0 \\
\hline
\end{tabular}


Table 3

Integrated experimental and calculated intensities - BMN

\begin{tabular}{|c|c|c|c|c|c|c|c|c|c|c|c|c|}
\hline \multirow[t]{2}{*}{$C_{\mathrm{g}}$} & \multicolumn{4}{|c|}{$25 \mathrm{~ns}$} & \multicolumn{4}{|l|}{$50 \mathrm{~ns}$} & \multicolumn{4}{|c|}{$100 \mathrm{~ns}$} \\
\hline & exp. & $l=1$ & $l=10$ & $I=100$ & exp. & $l=1$ & $l=10$ & $l=100$ & $\exp$. & $l=1$ & $I=10$ & $I=100$ \\
\hline 0.95 & & 4.7 & 5.8 & 5.8 & & 27.9 & 29.6 & 29.7 & & 66.7 & 59.1 & 59.5 \\
\hline 0.90 & 6.3 & 5.2 & 6.9 & 6.7 & 27.5 & 29.0 & 29.8 & 30.0 & 44.5 & 63.0 & 49.1 & 51.9 \\
\hline 0.85 & 5.6 & 5.8 & 7.5 & 6.2 & 27.6 & 29.7 & 29.1 & 30.0 & 19.0 & 58.8 & 43.7 & 56.6 \\
\hline 0.80 & 5.8 & 6.0 & 7.3 & 6.3 & 30.2 & 29.8 & 29.3 & 30.0 & 49.5 & 57.2 & 45.1 & 54.9 \\
\hline 0.70 & 7.1 & 6.0 & 6.0 & 5.7 & 29.0 & 30.0 & 29.9 & 29.4 & 51.9 & 58.0 & 57.7 & 50.9 \\
\hline 0.60 & 6.5 & & & & 28.9 & & & & 50.6 & & & \\
\hline
\end{tabular}

normalized intensity, i.e. summing over the emitted photons that decay from the naphthalene level to the ground state, and it is indicative of the extent of the energy transfer. In table 3 we do the same for the BMN emission.

The integrals up to 25 ns are of little interest. because the time curves up to this point reflect to a significant extent the laser signal. so no differentiation can be made. We thus concentrated on the 50 and 100 ns cases.

Notice that in all of the above we assumed that $\gamma=1$. The $[\mathrm{N}]$ solution, eq. (17), is rewritten as

$$
[\mathrm{N}]=\exp \left(-\int k_{\mathrm{N}} \mathrm{d} t\right) \exp \left(a \int \epsilon(1+b t) \mathrm{d} t\right)
$$

We see that $[\mathrm{N}]$ increases exponentially with $a$. Remembering that $a=n \gamma C_{\mathrm{s}} / t$, any uncertainty we have in $\gamma, n / t$, or $C_{\mathrm{s}}$ would carry exponentially to [N]. We saw that [47-49] $\gamma$ is less than 1.00 , ranging from $\approx 0.1$ to 0.5 within a factor of 2 or 3 . The problem of establishing the exact $C_{3}$ value was discussed previously, and using an "average" value. we may also be off the actual value by a factor of 2 or 3 . Finally. $n / t$, the number of steps per unit time. was derived from an uncertainty-type relation [49] and this may also be off by a factor of 2 or 3 .

To circumvent the above difficulties we made an adjustment to the calculated values in relation to the experimental results. At the crossover concentration region $\left(C_{\mathrm{g}}=0.80\right)$ all types of walks (i.e. different $l$ values) show about the same behavior, i.e. the same $\epsilon$. However, the experimental intensities do not agree with the calculated ones. The difference may be due to a combination of the three factors with significant uncertainties. These are $n / t, \gamma$, and $C_{s}$. which all combine to form $a=n \gamma C_{s}^{\prime} / r$. We therefore find an adjusted $a$. call it $a^{\prime}$. which when used in the differential equations produces a calculated value in agreement with the experiment for one given concentration value $C_{\underline{\underline{a}}}=$ 0.80 . Since we use the same $a^{\prime}$ for all guest concentrations. we preserve the relative magnitudes of the time-integrated intensities. The solutions to the differential equations plotted in fig. 4 include the adjusted factor $a^{\prime}$. as a function of $C_{\mathrm{a}}$ for $l=1.10$ and 100 . The results are shown in table 4. for integrated intensities to 50 and $100 \mathrm{~ns}$. for naphthalene, and in table 5 for BMN. Now a meaningful comparison with the experiment can be made. We plot the results of tables 4 and 5 in figs. 4 and 5. i.e. the total integrated intensities as a function of $C_{\mathrm{g}}$. for different coherence values.

From these plots we conclude that the $l=1$ curve shows the biggest deviation from experiment. while as $l$ increases the curves get closer to the experiment. Here. $l=100$ gives the best fit. Notice that we tested the adjusting factor $a^{\prime}$ over the total region of the "crossover" concentration (i.e. from guest concentration of $=0.75$ to 0.90 ). and we came up with practically the same results as in fig. 3. Finally, it should be pointed out that the values obtained from the adjusted factor are always within a factor of 2 of the unadjusted values.

The shape of the integrated intensity curve (fig. 4) shows a minimum around 0.90 . The reason for this is a bit complex. We notice (table 1 of ref. [33]) that the actual $B M N$ concentration is not constant in all crystals. bur steadily decreases. For 
Table 4

Reduced calculated intensities - naphthalene

\begin{tabular}{|c|c|c|c|c|c|c|c|c|}
\hline \multirow[t]{2}{*}{$\overline{C_{8}}$} & \multicolumn{4}{|l|}{$50 \mathrm{~ns}$} & \multicolumn{4}{|l|}{$100 \mathrm{~ns}$} \\
\hline & exp. & $l=1$ & $t=10$ & $l=100$ & exp. & $I=1$ & $t=10$ & $l=100$ \\
\hline 0.99 & 24.2 & 31.8 & 31.4 & 28.6 & 37.7 & 60.0 & 58.3 & 47.0 \\
\hline 0.95 & 18.6 & 28.5 & 27.6 & 20.1 & 24.6 & 46.7 & 43.2 & 24.0 \\
\hline 0.90 & 17.3 & 26.5 & 25.0 & 16.6 & 19.7 & 39.6 & 35.2 & 18.1 \\
\hline 0.85 & 19.9 & 26.5 & 25.0 & 16.6 & 24.6 & 35.1 & 32.4 & 23.6 \\
\hline 0.80 & 24.3 & 24.7 & 24.7 & 23.2 & 34.3 & 34.5 & 34.3 & 34.4 \\
\hline 0.75 & (25) & 25.0 & 27.0 & 29.0 & (35) & 35.4 & 41.4 & 54.0 \\
\hline 0.70 & 25.6 & 26.5 & 30.0 & 31.9 & 36.7 & 41.0 & 54.0 & 62.1 \\
\hline 0.65 & (25.3) & 28.2 & 32.0 & 31.7 & (36.7) & 46.7 & 62.0 & 61.8 \\
\hline 0.60 & 25.0 & 31.1 & 32.6 & 32.6 & 36.8 & 58.0 & 64.4 & 64.0 \\
\hline
\end{tabular}

example, from $C_{8}=0.99$ to 0.90 there is a factor of 10 difference in $C_{s}$, but from 0.90 to 0.40 there is only a factor of 4 . This uneven distribution (direct

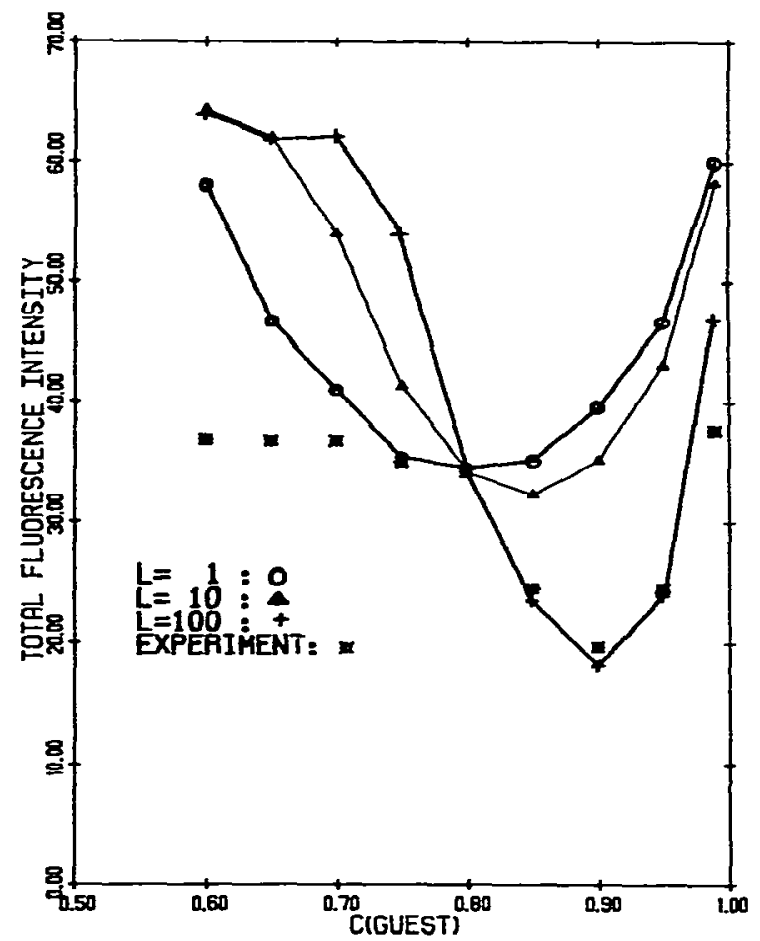

Fig. 4. Integrated naphthalene intensities as a function of $C_{8}$. The integrated intensities of table 4 are plotted as a function of $C_{\mathrm{g}}$. The theoretical curves for the tinree different coherence lengths are shown together with the experimental one. The naphthalene emission was monitored in this case.
Table 5

Reduced calculated intensities - BMN

\begin{tabular}{|c|c|c|c|c|c|c|c|c|}
\hline \multirow[t]{2}{*}{$C_{\mathrm{E}}$} & \multicolumn{4}{|c|}{$50 \mathrm{~ns}$} & \multicolumn{4}{|c|}{100 nsec } \\
\hline & exp. & $l=1$ & $t=10$ & $l=100$ & $\overline{\exp }$. & $l=1$ & $l=10$ & $l=100$ \\
\hline 0.95 & & 28.6 & 29.1 & 28.6 & & 64.6 & 62.5 & 41.2 \\
\hline 0.90 & 27.5 & 29.5 & 29.9 & 25.5 & 44.5 & 59.8 & 55.5 & 31.2 \\
\hline 0.85 & 27.6 & 29.9 & 29.9 & 28.3 & 49.0 & 55.3 & 52.1 & 40.1 \\
\hline 0.80 & 30.2 & 30.0 & 30.0 & 29.8 & 49.5 & 54.6 & 54.5 & 48.0 \\
\hline 0.75 & & 29.9 & 29.3 & 29.9 & & 55.5 & 61.3 & 46.0 \\
\hline 0.70 & 29.0 & 29.9 & 29.2 & 29.5 & 51.9 & 57.7 & 60.2 & 50.8 \\
\hline 0.60 & 28.9 & 28.5 & 28.0 & 28.0 & 50.6 & 64.2 & 62.6 & 62.5 \\
\hline
\end{tabular}

dependence on the host concentration) has a drastic effect on the fluorescence decay. Also, around $C_{\mathrm{g}}=\mathbf{0 . 8 0}$ the efficiency of the random walk drastically changes. The $I=100$ walker at $C_{\mathrm{g}}=0.90$ visits 66000 sites, but at 0.70 it visits only 1000

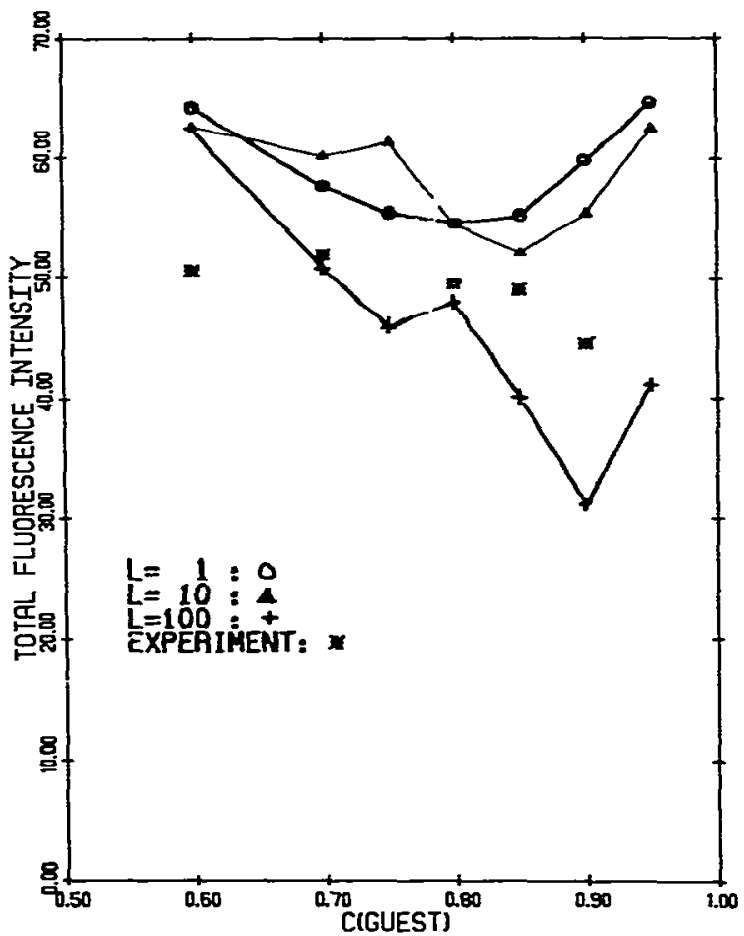

Fig. 5. Integrated $B M N$ intensities as a function of $C_{B}$. The integrated intensities of table 5 are plotted as a function of $C_{B}$. The theoretical curves for the three different coherence lengths are shown together with the experimental one. The BMN emission was monitored in this case. 
sites, a factor of 6 . It seems that these two opposing factors combined produce the resulting shape of fig. 4.

The above results are in good agreement with the results of the steady-state experiments we presented earlier [32,33]. The possible existence of coherence or "correlated walk" was first indicated there, and it is verified here in the following sense: The $l=1$ case presents the worst fit with the experiment, especially at high $C_{\mathrm{g}}$ and close to the critical dynamic percolation concentration. Because of all the uncertainties we do not report the value $l=100$ as the nominal solution to the problem. We merely state that partial coherence ("correlated walk") does describe our experiments in a consistent fashion.

\section{Discussion and conclusions}

We first performed "standard" steady-state fluorescence experiments [32,33] on the naphthalene system, and later the time-resolved tests described here. These are in reasonably good agreement with each other in the following way: (1) Based on the time-integrated intensities of the time-resolved data one gets results similar to those of the steady-state measurements [49]. (2) Neither result can be interpreted by simple incoherent hopping expressed via a simple random walk model (note that our "adjustable parameter" $a^{\prime}$ cannot account for the guest concentration dependence). (3) Using a "coherence" (correlation) parameter $l$ one can account for the observed concentration dependence of both steady-state and time-evolution experiments in a consistent way. We suggest that our correlation ("coherence") parameter does relate to partial coherence in terms of quantummechanical wave packets; however, this relationship is far from clear. This is our evidence for the possible existence of partial coherence. Our timeresolved spectra gave a good fit for "coherence" over $\approx 100$ lattice units. Our typical computer lattice size is $1000 \times 1000$ sites (for the two-dimensional case). On these lattices we performed tests for up to $I=250$. We noticed that for mixed crystals with guest concentrations up to $\approx 0.95$ any computer simulation with $l>100$ gives the same result as with $l=100$. because the heavy scattering by the host does not permit any differentiation between long and very long mean free paths. Therefore, our data would be the same had we used an $l=1000$ or greater (with a correspondingly larger lattice). This simply means that at $C_{g} \leqslant 0.95$ coherence is necessarily broken by the host scattering. Moreover, in the region $C_{\mathrm{g}} \leqslant 0.70$ the shallow guest traps. due to finite clusters. may cause additional scattering. Only for the neat (pure) crystal limit. or when closely approaching it, could higher $l$ values be tested in principle, but in this project we would not afford it. either computationally or experimentally. It is very difficult to prepare a crystal with no impurities or defects, in order to simulate the pure-crystal case. and test for larger coherence lengths. Therefore, our results do not preclude having $l \gg 100$ when exirarolated to a perfect latrice. We. therefore. conclude that we have tentatively established the existence of "coherence". at these temperatures, and mention that a value of $\approx 100$ lattice units is in good agreement with our experiments. This means that. extrapolated to the perfect pure naphthalene crystal, we get $l \geqslant 100$ for the temperature range studied (1.8-4.2 K).

A comparison with the absorption lineshapes of the pure naphthalene crystals in terms of exciton-phonon scattering was made in paper $I$ of this series [33]. It is obvious from our mixed-crystal energy-transport data that the host scattering overshadows the exciton-phonon scattering in crystais with $\approx 0.95$ guest concentration and below. This is qualitatively consistent with the significant broadening of the optical absorption lines at this and lower concentrations [56,57]. It is not clear to us yet how to make quantitative comparisons between the two kinds of data.

We have definitely established that no simple homogeneous kinetics model agrees with our data. Our exponential decay curves (for naphthalene) deviate so significantly from exponentiality that no model based on "rate constants" or non-dispersive diffusion can account for this. These deviations in our ternary crystals are much more drastic than usually observed for binary (lightly doped) crystals $[1,44]$. We note that there is still scepticism in the field concerning results from lightly doped 
crystals [58], due to possible complications from the supertrapping effects (e.g. the creation of large funnels $[1,44])$. We do not expect such complications to affect our measured guest concentration effects (see also ref. [47]) nor our large observed deviations from exponentiality. Whether dispersive diffusion models could account for our data remains to be seen. However. we do believe that for guest concentrations around 0.5 none of the above models will account for the very low temperature data. Here finite cluster states with energy mismatches equal or greater than $k T$ should dominate the kinetics. Because of the near confinement of guest excitons to finite guest clusters one should approach the interesting limit of two disjoint naphthalene exciton populations: (I) Excitons on clusters connected to a trap. (11) Excitons on clusters not connected to traps. Population (I) should decay in the time domain of picoseconds and from trapped BMN excitons. Population (II) should decay with practically the natural lifetime of the naphthalene singlet state. Evidence for such behavior has been given by Parson and Kopelman [29]. In this concentration regime our data are of lower quality but are in general agreement with such behavior. We can thus state that the observed exciton transport is only consistent with heterogeneous kinetics and that its full characterization is far from trivial.

The current models of energy transport in disordered materials [7-11] all seem to neglect the effects of exciton coherence. This ties-in with the yet unsolved aspects of localization versus delocalization in these systems $[13,26,30]$. We do not purport to answer here the basic question of partial coherence, but we present: (a) Experiments in well-defined systems that appear to be relevant to this question, whose interpretation will represent a challenge to future theories. (b) A parameterization via an oversimplified "coherence" parameter, which consists of a correlation of random walks and mimics a mean-free-path. In this model "coherence" (correlation) enhances exciton trapping under some conditions (pure crystal) and de-enhances it under others (high substitutional disorder). This seems to agree with the experimental results and may point towards the requirements of a satisfactory quantum-mechanical model.

\section{Appendix A: Proof of proportionality constant}

There are two channels of decay of an $\mathrm{N}$ excited state as shown in eq. (1), one with the rate constant $k_{\mathrm{N}}$ (with a probability of decay $Q$ ), and one with the time-dependent constant $k(t)$ (with a probability of decay $P$ ). The number of remaining excitons $N$ at any instance is proportional to the probability of non-decay of these excitons. Therefore, $N=(1-Q)(1-P)$. Note that at $t=0, Q=$ $0 . P=0, N=1$. The time-independent channel is a single exponential, so

$$
\begin{aligned}
& N=(1-P) \exp \left(-k_{\mathrm{N}} t\right) . \\
& \mathrm{d} N / \mathrm{d} t=-k_{\mathrm{N}}(1-P) \exp \left(-k_{\mathrm{N}} t\right) \\
& \quad-(\mathrm{d} P / \mathrm{d} t) \exp \left(-k_{\mathrm{N}} t\right) . \\
& (1 / N)(\mathrm{d} N / \mathrm{d} t)=-k_{\mathrm{N}}-(1 / 1-P)(\mathrm{d} P / \mathrm{d} t) .
\end{aligned}
$$

Utilizing eq. (1) we have

$$
(1 / N)(\mathrm{d} N / \mathrm{d} t)=-k_{\mathrm{N}}-k(t) \text {. }
$$

Combining these last two equations we have

$$
-k_{\mathrm{N}}-[1 /(1-P)](\mathrm{d} P / \mathrm{d} t)=-k_{\mathrm{N}}-k(t),
$$

which results in $k(t)=[1 /(1-P)](\mathrm{d} P / \mathrm{d} t)$, QED.

We note that for the limit $t \rightarrow \infty, P(t \rightarrow \infty) \rightarrow$ $P_{\infty}<1,(\mathrm{~d} P / \mathrm{d} t)_{t \rightarrow \infty} \rightarrow 0$, and thus $k(t \rightarrow \infty) \rightarrow 0$. The $\mathrm{N}$-decay thus becomes truly exponential, with the "natural" decay rate $k_{\mathrm{N}}$. Concomitantly the trapped exciton decay will also be exponential, with rate constant $k_{\mathrm{B}}$. We call this " the two-population limit" as there is no communication between the two and no coupling between the respective differential equations.

\section{References}

[1] R.C. Powell and Z.G. Soos, J. Luminescence, 11 (1975) 1.

[2] H.C. Wolf and H. Port, J. Luminescence, 12/13 (1976) 33.

[3] R. Silbey. Ann. Rev. Phys. Chem. 27 (1976) 203.

[4] 1.I. Abram and R.M. Hochstrasser, 1. Chem. Phys. 72 (1980) 3617 .

[5] R. Kopelman, in: Topics in applied physics, Vol. 15, ed. F.K. Fong (Springer, Berlin, 1976) p. 297.

[6] J. Klafter and J. Jortner, Chem. Phys. Letters 60 (1978) 5.

[7] A. Blumen and R. Silbey, J. Chem. Phys. 70 (1979) 3707.

[8] R.F. Loring and M.D. Fayer, Chem. Phys. 70 (1982) 139: R.F. Loring, H.C. Andersen and M.D. Fayer, J. Chem. Phys. 76 (1982) 2015; 
C.R. Gochanour, H.C. Andersen and M.D. Fayer, J. Chem. Phys. 70 (1979) 4254.

[9] R.A. Auerbach, G.W. Robinson and R.W. Zwanzig, J. Chem. Phys. 72 (1980) 3528.

[10] S.W. Haan and R. Zwanzig, J. Chem. Phys. 68 (1978) 1879.

[11] D.L. Huber, Phys. Rev. B 20 (1979) 2307.

[12] A. Blumen and J. Manz. J. Chem. Phys. 71 (1979) 4694.

[13] A. Blumen, J. Klafter and R. Silbey, J. Chem. Phys. 72 (1980) 5320.

[14] J. Klafter and R. Silbey, J. Chem. Phys. 72 (1980) 843.

[15] J. Klafter and R. Silbey, J. Chem. Phys. 72 (1980) 849.

[16] K. Allinger and A. Blumen, J. Chem. Phys. 72 (1980) 4608.

[17] J. Klafter and R. Silbey, Phys. Rev. Letters 44 (1980) 55.

[18] R.W. Munn and R. Silbey, J. Chem. Phys. 68 (1978) 2439.

[19] A. Blumen and R. Silbey. J. Chem. Phys. 70 (1979) 3707.

[20] R. Silbey and R.W. Munn, J. Chem. Phys. 72 (1980) 2763.

[21] A. Blumen, J. Chem. Phys. 72 (1980) 2632.

[22] K. Godzik and J. Jortner, J. Chem. Phys. 72 (1980) 4171.

[23] A. Blumen, J. Chem. Phys. 71 (1979) 4694.

[24] A. Blumen and J. Manz, J. Chem. Phys. 74 (1981) 6926.

[25] A.H. Francis and R. Kopelman, in: Topics in applied physics, Vol. 49, eds. W.M. Yen and P.M. Selzer (Springer, Berlin, 1980) p. 241.

[26] R. Kopelman, in: Modern problems in solid state physics. Vol. 4. Spectroscopy and excitation dynamics of condensed molecular systems, eds. V.M. Agranovich and R.M. Hochstrasser (North-Holland, Amsterdam, 1983).

[27] D.M. Burland and A.H. Zewail, in: Advances in chemical physics, Vol. 40, eds. I. Prigogine and S.A. Rice (Wiley, New York, 1979).

[28] S.D. Colson, S.M. George, T. Keys and V. Vaida, J. Chem. Phys. 67 (1977) 4941.

[29] R.P. Parson and R. Kopelman. Chem. Phys. Letters 87 (1982) 528

[30] S.T. Gentry and R. Kopelman, J. Chem. Phys. 78 (1983) 373.

[31] R.M. Pearlstein. Photochem. Photobiol. 35 (1982) 835.

[32] P. Argyrakis and R. Kopelman, J. Chem. Phys. 66 (1977) 3301.

[33] P. Argyrakis and R. Kopelman, Chem. Phys. 57 (1981) 29.

[34] E.W. Montroll, Proceedings of the 16th Symposium on Applied Mathematics (1964) 193.

[35] P. Argyrakis and R. Kopelman, Phys. Rev. B22 (1980) 1830.

[36] P. Argyrakis, Phys. Rev. B27 (1983) 1355.

[37] P. Argyrakis, E.M. Monberg and R. Kopelman, Chem. Phys. Letters 36 (1975) 349.

[38] R. Kopelman, in: Excited states, Vol. 2. ed. E.C. Lim (Academic Press, New York, 1975).
[39] R. Kopelman and P. Argyrakis. J. Chem. Phys. 72 (1980) 3053.

[40] J. Hoshen and R. Kopelman, J. Chem. Phys. 65 (1976) 2817.

[41] P. Argyrakis and R. Kopelman. J. Theoret. Biol. 73 (1978) 205.

[42] C.E. Swenberg and N.E. Ciescintov, in: Organic molecular photophysics, Vol. 1, ed. J.B. Birks (Wiley, New York. 1973):

N.E. Geacintov and C.E. Swenberg. in: Luminescence spectroscopy, ed. M.D. Lumb (Academic Press. New York. 1978).

[43] S. Fujiyara, T. Nakajama and N. Itoh. Phys. Stat. Sol. $78 b$ (1976) 519.

[44] H. Auweter. A. Braun. U. Mayer and D. Schmid. Z. Naturforsch. 34a (1979) 761.

[45] P.W. Klymko and R.P. Parson, private communication.

[46] R. Kopelman. J. Hoshen. J.S. Neuhouse and P. Argyrakis. J. Stat. Phys. 30 (1983) 355.

[47] P. Argyrakis. D. Hooper and R. Kopelman. J. Phys. Chem. 87 (1983) 1467.

[48] E.M. Monberg. Doctoral Dissertation. University of Michigan (1977).

[49] P. Argyrakis, Doctoral Dissertation, University of Michigan (1979).

[50] M.R. Caprini. S. Cohn-Sfetchi and A.Mf. Manof. IEEE Trans. Audio and Flectroacoustics. AU-18 (1970) 389.

[51] R. Bracewell, The Fourier transform and its applications (McGraw-Hill, New York, 1965).

[52] W.S. Stanley. Digital signal processing (Reston. New York. 1975).

[53] G. Horlick, Anal. Chem. 43 No. 8 (1971) 61A.

[54] J.W. Cooley and J.W. Tuckey, Math. Comput. 19 (1965) 297.

[55] E.O. Brigham. The fast Fourier transform (Prentice-Hall. Englewood Cliffs. 1974).

[56] H.-K. Hong and G.W. Robinson. J. Chem. Phys. 52 (1970) 825.

[57] H.-K. Hong and G.W. Robinson. J. Chem. Phys. 54 (1971) 1369.

[58] V.M. Kenkre and D. Schmid. Conference on Energy transfer in Organic Solids. University of Rechester. 17 September 1982 .

[59] A. Blumen and G. Zumofen. J. Chem. Phys. 75 (1981) 992.

[60] G. Zumofen and A. Blumen. J. Chem. Phys. 76 (198?) 3713.

[61] S.T. Gentry and R. Kopelman. Phys. Rev. B27 (1983) 2579.

[62] A. Braun, H. Pfisterer and D. Schmid. J. Luminescence 17 (1978) 15. 